\title{
The impact of intolerance of uncertainty and cognitive behavioural instructions on safety learning
}

Article

Published Version

Creative Commons: Attribution 4.0 (CC-BY)

Open Access

Wake, S., van Reekum, C. M., Dodd, H. and Morriss, J. (2020) The impact of intolerance of uncertainty and cognitive behavioural instructions on safety learning. Cognitive Therapy and Research, 44 (5). pp. 931-942. ISSN 0147-5916 doi: https://doi.org/10.1007/s10608-020-10113-4 Available at https://centaur.reading.ac.uk/91074/

It is advisable to refer to the publisher's version if you intend to cite from the work. See Guidance on citing.

Published version at: http://dx.doi.org/10.1007/s10608-020-10113-4

To link to this article DOI: http://dx.doi.org/10.1007/s10608-020-10113-4

Publisher: Springer

All outputs in CentAUR are protected by Intellectual Property Rights law, including copyright law. Copyright and IPR is retained by the creators or other copyright holders. Terms and conditions for use of this material are defined in the End User Agreement.

www.reading.ac.uk/centaur 
Central Archive at the University of Reading

Reading's research outputs online 


\title{
The Impact of Intolerance of Uncertainty and Cognitive Behavioural Instructions on Safety Learning
}

\author{
Shannon Wake ${ }^{1} \cdot$ Carien M. van Reekum ${ }^{1} \cdot$ Helen Dodd $^{1} \cdot$ Jayne Morriss $^{1}$ (I)
}

(c) The Author(s) 2020

\begin{abstract}
Background Difficulty updating threat associations to safe associations has been observed in individuals who score high in self-reported Intolerance of Uncertainty (IU). Here we sought to determine whether an instruction based on fundamental principles of Cognitive Behavioural Therapy could promote safety learning in individuals with higher levels of IU, whilst controlling for self-reported trait anxiety (STICSA).

Methods We measured skin conductance response, pupil dilation and expectancy ratings during an associative threat learning task in which participants either received a cognitive behavioural instruction or no instruction prior to threat extinction $(n=92)$.

Results Analyses revealed that both self-reported IU and STICSA similarly predicted differences in skin conductance response. Only individuals with lower IU/STICSA in the cognitive behavioural instruction condition displayed successful safety learning via skin conductance response.

Conclusions These initial results provide some insight into how simple cognitive behavioural instructions combined with exposure are applied differently in individuals with varying levels of self-reported anxiety. The results further our understanding of the role of basic cognitive behavioural principles and self-reported anxiety in safety learning.
\end{abstract}

Keywords Anxiety · Intolerance of uncertainty · Conditioning · Extinction · CBT · Psychophysiology

\section{Introduction}

The ability to learn and update threat and safety associations is essential for maintaining health and well-being (Milad and Quirk 2012; Shin and Liberzon 2009). Learning to associate cues with threat can protect us from dangerous situations. However, when a cue no longer signals threat, it is adaptive to update the previously learned association between cue-threat to cue-safety. Through threat conditioning, an organism can associate a neutral cue (conditioned stimulus,

Electronic supplementary material The online version of this article (doi:https://doi.org/10.1007/s10608-020-10113-4) contains supplementary material, which is available to authorized users.

Jayne Morriss

j.e.morriss@ reading.ac.uk

1 Centre for Integrative Neuroscience and Neurodynamics, School of Psychology and Clinical Language Sciences, University of Reading, Earley Gate, Whiteknights Campus, Reading RG6 6AH, UK e.g., visual stimulus, such as a shape) with an aversive outcome (unconditioned stimulus (US), e.g., loud noise, electric shock). Repeated presentations of a neutral cue with an aversive outcome can result in threat responding to the cue alone (conditioned response). Moreover, by repeatedly presenting the learned threat cue without the aversive outcome, the learned threat association can be mitigated, as indexed by a reduction in threat responding. This process is known as threat extinction (Milad and Quirk 2012). During threat extinction, a reduction in responsivity to the learned threat cue over time is believed to reflect changes in contingency beliefs and harm expectancy (Hofmann 2008). However, uncertainty about changes in contingency from threat to safety might prolong, or prevent, the learning of new safety associations (Bouton 2002), potentially resulting in dysfunctional fears and consequently affecting an individual's quality of life.

Previous research has shown that physiological responses are elevated and sustained to cues that no longer signal threat in anxiety and stress disorders, suggesting impaired threat extinction (Blechert et al. 2007; Michael et al. 2007; Milad 
et al. 2008, 2009). Intolerance of uncertainty (IU) (Freeston et al. 1994) has been defined as "an individual's dispositional incapacity to endure the aversive response triggered by the perceived absence of salient, key, or sufficient information, and sustained by the associated perception of uncertainty" (Carleton 2016b). Recent work has begun to examine how IU affects safety learning and has demonstrated that higher IU is associated with reduced safety learning, indexed by greater skin conductance responding to cues that no longer signal threat throughout extinction (Morriss et al. 2015, 2016; Morriss, 2019; Morriss and van Reekum 2019). Further research has shown that individuals with higher IU are more prone to experience spontaneous recovery of learned threat (Dunsmoore et al. 2015; Lucas et al. 2018).

Threat extinction is inherently uncertain, as the change in contingency from threatening to safe isn't obvious. The uncertainty experienced during threat extinction is thought to maintain the conditioned response in individuals with high IU (Morriss and van Reekum 2019; Morriss et al. 2019a, b). This explanation is in line with a current definition of IU, where the omission of information sustains the perception of uncertainty (Carleton 2016b, p. 31). Importantly, in a recent study it was shown that giving contingency instructions before threat extinction (i.e., revealing the new safe outcome) enhanced safety learning in individuals with high IU (Morriss and van Reekum 2019). These results further support the notion that it is the perceived uncertainty during threat extinction that disrupts safety learning in individuals with high IU. Given that IU is transdiagnostic (Carleton et al. 2016a), and that current exposure therapies are based on principles of associative learning (Craske et al. 2014), we can speculate that IU may be one of the reasons why some individuals may take longer to benefit from exposure therapies. Therefore, examining the circumstances under which safety learning may be promoted in individuals with high IU will reveal crucial information to facilitate the development of clinical interventions that can target IU across disorders with an anxiety component (Craske et al. 2014; Knowles and Olatunji 2019).

According to cognitive theory of anxiety, the re-evaluation of the probability and the costs of the feared outcome should lead to a corresponding decrease in worry and anxiety about the potential outcome. Hence, one of the aims of cognitive therapy is to help anxious clients re-evaluate both the probability that the feared outcome will take place and the consequences should it occur (Robichaud et al. 2019). Research has examined whether IU can be targeted in Cognitive Behaviour Therapy (CBT) for anxiety disorders and findings show promise. Dugas and colleagues (e.g., Dugas and Ladouceur 2000; Dugas et al. 2003, 2010) have developed a cognitive-behavioural intervention designed to specifically target IU as part of a global treatment for generalised anxiety disorder (GAD). This treatment has been shown to effectively reduce IU and other symptoms, such as worry and depression in individuals with GAD (Bomyea et al. 2015). Also, CBT treatments for GAD that do not specifically target IU have demonstrated a reduction of IU across treatment (e.g., Boswell et al. 2013; Hewitt et al. 2009; van der Heiden et al. 2012). Further work also demonstrates that changes in IU are associated with symptom relief in social anxiety disorder and GAD across three different CBT protocols (McEvoy and Erceg-Hurn 2015). There is therefore good reason to believe that IU is malleable, with a transdiagnostic and trans-therapy construct. However, the processes by which IU changes in therapy remain relatively unknown and research is needed to understand the mechanisms of change across different treatment interventions (Shihata et al. 2016).

Treatments for anxiety and stress-based disorders commonly combine both CBT and exposure therapy (Dugas and Robichaud 2007; Simos and Hofmann 2013). To examine whether the combination of CBT and exposure-based principles promote safety learning in individuals with high IU, we developed a lab-based extinction task where an instruction derived from CBT concepts was presented before the threat extinction phase. This instruction was centred around cognitive aspects of CBT, by encouraging participants to re-evaluate their predictions about the uncertainty of the CS and US pairing. We measured skin conductance responses and expectancy ratings whilst participants underwent threat acquisition and extinction. We used an aversive sound as an unconditioned stimulus and visual shapes as conditioned stimuli, similar to previous conditioning research (Morriss et al. 2015, 2016; Neumann and Waters 2006). We used a 50\% reinforcement rate during acquisition to sustain conditioning during extinction (Grady et al. 2016; Leonard 1975). Participants were assigned to either a cognitive behavioural (CB) instruction or no instruction condition. Prior to threat extinction, participants in the CB instruction condition were encouraged to pay attention to the cues (blue and yellow squares) and assess whether a sound would follow. They were then instructed to try to use this information to help them determine what to expect the next time they saw a square. Participants in the no instruction condition were only told to pay attention to the squares and sounds prior to the threat extinction phase. To ensure that an equal balance of low and high IU individuals were assigned to each condition, another member of the lab matched participants across conditions based on self-reported IU score.

We hypothesised that during threat acquisition, all participants regardless of condition would exhibit greater conditioned responding indexed by larger skin conductance response magnitudes and higher expectancy ratings to the learned threat $(\mathrm{CS}+)$ versus safety $(\mathrm{CS}-)$ cues. Based on previous research, we predicted that higher IU, relative to lower IU in the no instruction condition would be associated 
with greater conditioned responding during the late part of the extinction learning phase (i.e., last $8 \mathrm{CS}+/ \mathrm{CS}-$ trials) (Morriss et al. 2015, 2016). If a CB instruction promotes safety learning in individuals with higher levels of IU, then higher IU in the CB instruction condition should be associated with reduced conditioned responding during the late part of extinction learning (i.e., last $8 \mathrm{CS}+/ \mathrm{CS}-$ trials) compared to those with higher IU in the no instruction condition.

In line with previous work (for discussion see Morriss et al. 2016) we tested the specificity of IU effects by controlling for trait anxiety, in this study assessed by the State-Trait Inventory for Cognitive and Somatic Anxiety (STICSA) (Ree et al. 2008). We selected the STICSA because it is thought to be a purer measure of anxiety, compared to other trait anxiety measures which also feature depressive symptomology (Grös et al. 2007).

The details of the current study (i.e., hypotheses, data collection, analysis plans and rules for data exclusion) were preregistered to the Open Science Framework before study commencement (https://osf.io/zv96d).

\section{Method}

\section{Participants}

A community sample of 95 participants were recruited from the University of Reading and local area through the use of advertisements and word of mouth $(M$ age $=24.4$ years, $S D=4.41$ years, range $=18-35$ years; Sex: 67 female, 28 male; Ethnicity: 61 White, 10 Asian, 3 Middle Eastern/Arab, 2 Black, 1 Mixed, and 14 not specified; Sexual orientation: 63 Heterosexual, 7 Sexual minorities (homosexual, bisexual, pansexual), 25 not specified). There were 2 participants that withdrew and 1 participant did not understand the task instructions, leaving a sample of 92 participants.

Participants were recruited if they were between 18 and 35 years of age. No other exclusion criteria were used for recruitment. Recruitment was not restricted based on IU score. Participants were paid $£ 5$ on the day of testing to remunerate them for their time. The procedure was reviewed and accepted by the University of Reading Research Ethics Committee. Participants provided written informed consent.

The initial power analyses estimated 92 participants. However, we realised that it included an incorrect $f$ value. Therefore, we updated the power analyses to the following. The sample size of this study was based on a power analysis using the average effect size $\left(\eta^{2} \mathrm{p}=0.16\right)$ taken from Stimulus $\times$ Time $\times$ IU interactions for SCR magnitude from five previous experiments ( $4 / 5$ with significant effects of IU) (Morriss et al. 2015, 2016; Morriss and van Reekum 2019). The following parameters were used: effect size $f=0.43$ (converted from $\eta^{2} \mathrm{p}=0.16$ ), $\alpha$ error probability $=0.05$,
Power (1- error probability) $=0.95$, number of groups $=2$ (no instruction, CB instruction), numerator $d f=1$, number of covariates $=2$ (IU, STICSA). The total sample size required was $n=73$. Based on the updated power analysis, we oversampled.

We intended to conduct analyses using multilevel models and enter IU as a continuous predictor variable. However, we based the power analysis on a repeated measures withinbetween interaction ANCOVA design because there is no agreed method for calculating power and estimating sample size for multilevel models (MLM) (Peugh 2010; Snijders 2005).

\section{Procedure}

Upon arrival at the laboratory, participants were informed about the experimental procedures. Participants were seated in the testing booth and asked to complete a consent form and a series of questionnaires (see below) on the computer screen. To ensure that an even number of high and low participants were allocated to each condition, a second research assistant assigned each participant to a condition based on their IU score at the beginning of the experiment. Based on previous research, low IU was defined as $<$ average score of 65 and high IU was defined as $=>65$ (Morriss and van Reekum 2019).

Next, the eyetracker was mounted upon the participants' head and physiological sensors were attached to the participants' left hand. Before the task started, participants were instructed to: (1) maintain attention to the task by looking at the coloured squares and listening to the sounds, which may be unpleasant, (2) respond to the expectancy rating scales that follow the end of each block of trials, using number keys on the keyboard with their dominant hand and (3) to stay as still as possible. Pupil dilation, skin conductance response and behavioural ratings were recorded whilst the conditioning task (see "Conditioning Task" below for details) was presented on a computer screen. The experimental session lasted approximately $30 \mathrm{~min}$ in total.

\section{Conditioning Task}

The conditioning task was designed using E-Prime 2.0 software (Psychology Software Tools Ltd, Pittsburgh, PA) (identical to previous work, Morriss, 2019). Visual stimuli were presented at a $75 \mathrm{~Hz}$ refresh rate on a 21 inch colour monitor (DiamondPro, Sony). Participants sat approximately $60 \mathrm{~cm}$ from the screen. Visual stimuli were blue and yellow squares with visual angles of $6.16^{\circ} \times 9.07^{\circ}$. The aversive sound stimulus was presented through headphones and consisted of a female scream that had been used in previous experiments (Morriss et al. 2015, 2016). The volume of the sound was 
standardized across participants by using fixed volume settings on the presentation computer and verification by an audiometer prior to each session $(90 \mathrm{~dB})$.

The task comprised of two threat learning phases: acquisition and extinction. Both acquisition and extinction phases consisted of two blocks each. During acquisition, one of the coloured squares (blue or yellow) was paired with the aversive $90 \mathrm{~dB}$ sound $50 \%$ of the time (CS+), whilst the other square (yellow or blue) was presented alone (CS-). A 50\% pairing rate was used to maximise the unpredictability of the CS+/US contingency. Conditioning contingencies were counterbalanced, with half of participants receiving the blue square paired with the US and the other half of participants receiving the yellow square paired with the US.

Prior to extinction, participants in the CB instruction condition were presented the following instruction: "In the next phase of the experiment we want you to pay attention to the squares and whether the sounds follows. Try to use this information to help you know what to expect next time you see another square. Please keep this in mind throughout this phase of the experiment". Participants were asked to confirm that they fully understood the statement before moving on to the next phase of the experiment. Prior to extinction, participants in the no instruction condition were presented with the following instruction: "The next phase of the experiment will begin shortly. Please pay attention to the squares and sounds." Participants were asked to confirm that they fully understood the statement before moving on to the next phase of the experiment. During threat extinction, both the blue and yellow square were presented in the absence of the US; this was true for both conditions, CB instruction and no instruction.

The acquisition phase consisted of 24 trials $(6 \mathrm{CS}+$ paired, $6 \mathrm{CS}+$ unpaired, $12 \mathrm{CS}-$ ). The extinction phase comprised of 32 trials (16 CS+ unpaired and 16 CS-), where "early" was defined as the first $8 \mathrm{CS}+/ \mathrm{CS}-$ trials and "late" was defined as the last $8 \mathrm{CS}+/ \mathrm{CS}-$ trials. Blocks of trials in acquisition consisted of 12 trials and in extinction consisted of 16 trials ( 2 blocks per phase). Experimental trials were pseudo-randomized such that the first trial of acquisition was always paired and then after all trial types were randomly presented within blocks. In acquisition participants could either receive a maximum of $2 \mathrm{CS}+$ paired, $2 \mathrm{CS}+$ and 3 $\mathrm{CS}-$ 's in a row. In extinction participants could receive a maximum of $4 \mathrm{CS}+$ 's or CS- 's in a row. The coloured squares were presented for a total of $4000 \mathrm{~ms}$. The aversive sound lasted for $1000 \mathrm{~ms}$ and terminated with the offset of the paired square (CS+). Subsequently, a blank screen was presented for 6000-8800 ms (Morriss et al. 2019a, b; Morriss and van Reekum 2019).

After trial 12, 24, 40, and 56, participants were asked to rate how much they expected the blue square and the yellow square to be followed by the sound stimulus. The scales ranged from 1 ("Don't Expect") to 9 ("Do Expect"). All participants were presented with two other 9-point Likert scales at the end of the experiment. Participants were asked to rate: (1) the valence and (2) the arousal elicited by the sound stimulus. These scales ranged from 1 (Valence: very negative; Arousal: calm) to 9 (Valence: very positive; Arousal: excited).

\section{Questionnaires}

To assess IU and trait anxiety, we administered the Intolerance of Uncertainty Scale (Freeston et al. 1994) and STICSA questionnaires (Ree et al. 2008). The IU measure consists of 27 items that are rated on a 5-point Likert scale. The STICSA consists of 21 items that are rated on a 4-point Likert scale.

\section{Ratings Scoring}

Rating data were reduced for each participant by calculating their average responses for each experimental condition (Acquisition CS+; Acquisition CS-; Extinction CS+Early; Extinction CS- Early, Extinction CS+Late; Extinction CS- Late) using the E-Data Aid tool in E-Prime (Psychology Software Tools Ltd, Pittsburgh, PA).

Two participants' ratings data were lost due to a computer copying error, leaving 90 participants with useable ratings data.

\section{Skin Conductance Acquisition and Scoring}

Physiological recordings were obtained using AD Instruments (AD Instruments Ltd, Chalgrove, Oxfordshire) hardware and software. Electrodermal activity was measured with dry MLT116F silver/silver chloride bipolar finger electrodes that were attached to the distal phalanges of the index and middle fingers of the non-dominant hand. A low constant-voltage AC excitation of $22 \mathrm{mVrms}$ at $75 \mathrm{~Hz}$ was passed through the electrodes, which were connected to a ML116 GSR Amp, and converted to DC before being digitized and stored. An ML138 Bio Amp connected to an ML870 PowerLab Unit Model 8/30 amplify the skin conductance signal, which was digitized through a 16-bit A/D converter at $1000 \mathrm{~Hz}$. The electrodermal signal was converted from volts to microSiemens using AD Instruments software (AD Instruments Ltd, Chalgrove, Oxfordshire).

Skin conductance responses were marked using ADinstruments software (AD Instruments Ltd, Chalgrove, Oxfordshire) and extracted using Matlab R2017a software (The MathWorks, Inc., Natick, Massachusetts, United States). CS+ unpaired and CS- trials were included in the analysis, but $\mathrm{CS}+$ paired trials were discarded to avoid sound confounds. Skin conductance responses (SCR) were scored 
when there is an increase of skin conductance level exceeding 0.03 microSiemens (Dawson et al. 2000). The amplitude of each response was scored as the difference between the onset and the maximum deflection prior to the signal flattening out or decreasing. SCR onsets and respective peaks were counted if the SCR onset was within 0.5-3.5 s (CS response) following CS onset (Morriss et al. 2018a, b). Trials with no discernible SCRs were scored as zero. SCR magnitudes were square root transformed to reduce skew and z-scored within-subjects to control for interindividual differences in skin conductance responsiveness (Ben-Shakhar, 1985). SCR magnitudes were calculated from remaining trials by averaging SCR-transformed values for each condition (Acquisition $\mathrm{CS}+$; Acquisition CS-; Extinction CS+ Early; Extinction CS- Early; Extinction CS+ Late; Extinction CS- Late). We defined non-responders as those who responded to $10 \%$ or less of the total CS+ unpaired and CS- trials across acquisition and extinction phases (50 trials total) (Xia, Dymond, Lloyd \& Vervliet, 2017). Therefore participants were excluded if they had 5 trials or less with valid SCR responses (for distributions of skin conductance responding, please see Supplementary Material). Eight non-responders were excluded from the SCR analyses, leaving 86 participants with useable SCR data.

\section{Pupil Dilation Acquisition and Scoring}

Pupil dilation was recorded using an Eyelink II eye-tracker with a sampling rate of $250 \mathrm{~Hz}$ (SR Research). Head movements were constrained with a chin-rest at a viewing distance of $60 \mathrm{~cm}$. The eyetracker was calibrated using a standard 3-point grid at the start of the experiment and operated in pupil and corneal reflection recording mode.

Pupil dilation was extracted using Matlab R2017a software (The MathWorks, Inc., Natick, Massachusetts, United States). CS+ unpaired and CS- trials were included in the analysis, but CS+ paired trials were discarded to avoid sound confounds. Pupil dilation was averaged for $4000 \mathrm{~ms}$ following CS onset. These data were baseline corrected by subtracting $1000 \mathrm{~ms}$ preceding each CS onset from a blank screen (Lonsdorf et al. 2017). Following this pupil dilation data were z-scored to control for interindividual differences in pupil dilation size (Leuchs et al. 2019). Trials were averaged per stimulus type for each participant resulting in the following conditions (Acquisition CS+; Acquisition CS-; Extinction CS+ Early; Extinction CS- Early; Extinction CS+ Late; Extinction CS- Late).

Due to problems calibrating the eyetracker, four participants' data were not recorded, leaving 88 participants with useable pupil dilation data.

\section{Ratings, SCR Magnitude and Pupil Dilation Analysis}

The analyses were conducted using the mixed procedure in SPSS 25.0 (SPSS, Inc; Chicago, Illinois). We conducted separate MLMs for expectancy ratings, SCR magnitude and pupil dilation during acquisition and Extinction. For expectancy ratings and SCR magnitude during the acquisition phase we entered Instruction Type (CB instruction and no instruction) and Stimulus (CS+, CS-) at level 1 and individual subjects at level 2. For expectancy ratings and SCR magnitude during the extinction phase we entered Instruction Type (CB instruction and no instruction), Stimulus (CS+, CS-) and Time (Early: first 8 CS+/CS- trials, Late: last 8 CS+/CS- trials) at level 1 and individual subjects at level 2. We included the following individual difference predictor variables in the multilevel models: IU and STICSA.

In all models, we used a diagonal covariance matrix for level 1. Random effects included a random intercept for each individual subject, where a variance components covariance structure was used. Fixed effects include Instruction Type, Stimulus, and Time. We used a maximum likelihood estimator for the multilevel models. We corrected post-hoc tests for multiple comparisons using the Benjamini-Hochberg False Discovery Rate procedure (Benjamini and Hochberg, 1995). The corrected significance values were: extinction ratings, $p<0.024$; extinction SCR magnitude, $p<0.016$; extinction pupil dilation, $p<0.003$. The significance values for the acquisition phase were not corrected as there was only one test (i.e., CS+vs. CS-).

In the MLMs with the two predictor variables (IU, STICSA), a significant interaction with one variable but not the other suggests specificity. Based on prior work, we expected such specificity for IU, but we also explored interactions with STICSA, given extant findings with trait anxiety in the conditioning literature (Lonsdorf et al. 2017). Where a significant interaction was observed with IU (or STICSA), we performed follow-up pairwise comparisons on the estimated marginal means of the relevant conditions estimated at specific IU values of + or - $1 \mathrm{SD}$ of mean IU, adjusted for STICSA (or IU). Similar analyses have been published elsewhere (Morriss et al. 2016, 2018b).

\section{Results}

For descriptive statistics see Table 1.

\section{Questionnaires}

The anxiety measures were normally distributed (skewness and kurtosis values between 0.3 and 0.7$)$ : IU $(M=63.17$, $S D=21.18$, range $=28-113, \alpha=0.95)$; STICSA $(M=38.67$, 
Table 1 Summary of means (SD) for each dependent measure as a function of condition (CS+ and CS-), separately for acquisition, early extinction and late extinction

\begin{tabular}{|c|c|c|c|c|c|c|}
\hline \multirow[t]{2}{*}{ Measure } & \multicolumn{2}{|c|}{ Acquisition } & \multicolumn{2}{|c|}{ Early extinction } & \multicolumn{2}{|c|}{ Late extinction } \\
\hline & $\mathrm{CS}+$ & CS- & $\mathrm{CS}+$ & CS- & $\mathrm{CS}+$ & CS- \\
\hline Expectancy rating (1-9) & $\begin{array}{l}6.59 \\
(1.99)\end{array}$ & $\begin{array}{l}2.31 \\
(1.88)\end{array}$ & $\begin{array}{l}4.50 \\
(2.63)\end{array}$ & $\begin{array}{l}1.88 \\
(1.53)\end{array}$ & $\begin{array}{l}3.20 \\
(2.27)\end{array}$ & $\begin{array}{l}1.97 \\
(1.98)\end{array}$ \\
\hline $\begin{array}{l}\text { Square root transformed and } \\
\text { Z-scored SCR magnitude }(\sqrt{ } \mu \mathrm{s})\end{array}$ & $\begin{array}{l}0.20 \\
(0.50)\end{array}$ & $\begin{array}{l}-0.03 \\
(0.29)\end{array}$ & $\begin{array}{l}0.07 \\
(0.33)\end{array}$ & $\begin{array}{l}-0.10 \\
(0.32)\end{array}$ & $\begin{array}{l}0.06 \\
(0.37)\end{array}$ & $\begin{array}{l}-0.13 \\
(0.39)\end{array}$ \\
\hline Z-scored pupil dilation $(\Delta \mathrm{mm})$ & $\begin{array}{l}0.14 \\
(0.48)\end{array}$ & $\begin{array}{l}0.06 \\
(0.39)\end{array}$ & $\begin{array}{l}0.05 \\
(0.40)\end{array}$ & $\begin{array}{l}-0.11 \\
0.38\end{array}$ & $\begin{array}{l}-0.04 \\
0.41\end{array}$ & $\begin{array}{l}-0.09 \\
0.44\end{array}$ \\
\hline
\end{tabular}

SCR magnitude $(\sqrt{ } \mu \mathrm{S})$, square root transformed and z-scored skin conductance magnitude measured in microSiemens. Z-scored pupil dilation $(\Delta \mathrm{mm})$ measured in delta millimetres
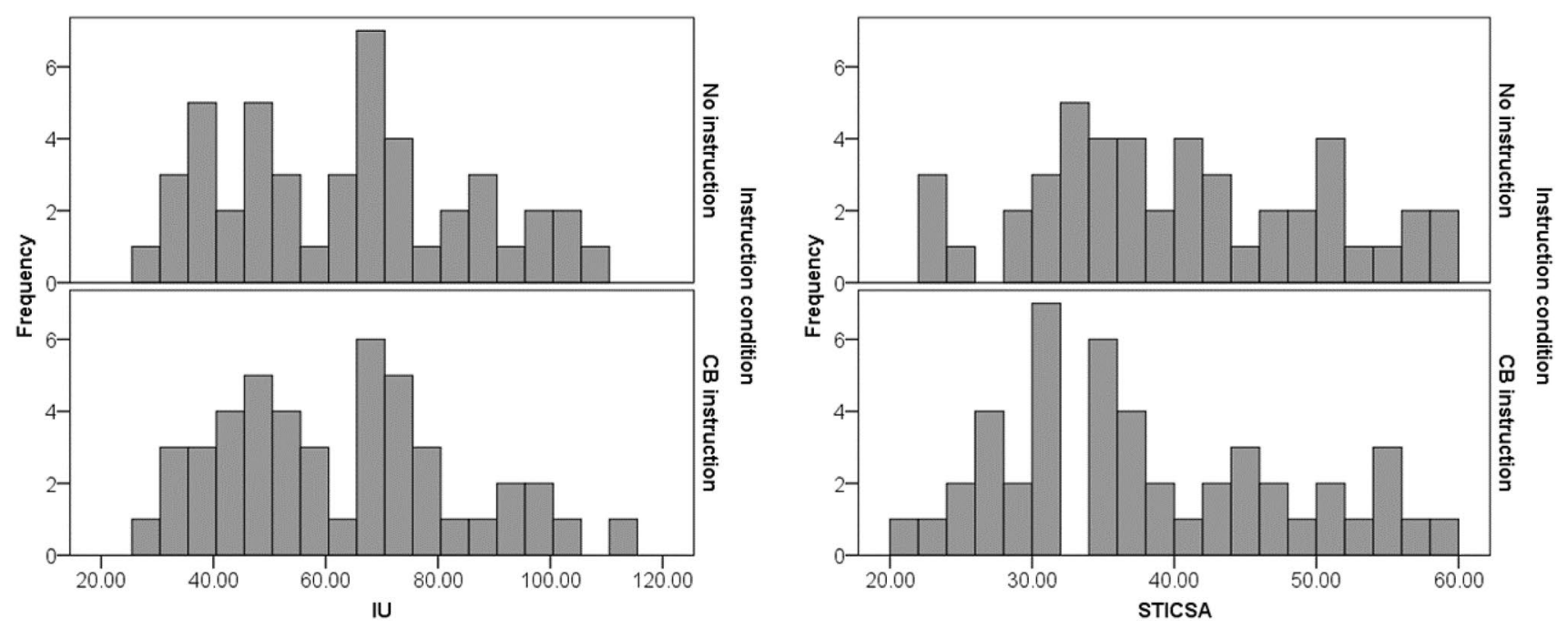

Fig. 1 Histograms depicting the distribution of IU and STICSA self-report scores per instruction condition

$S D=10$, range $=21-58, \alpha=0.89)$. The no instruction and $\mathrm{CB}$ instruction conditions had a similar range of scores for the IU and STICSA measures (no instruction IU: $\mathrm{M}=63.63$, $\mathrm{SD}=21.77$; $\mathrm{CB}$ instruction IU: $\mathrm{M}=62.71, \mathrm{SD}=20.81$; no instruction STICSA: $\mathrm{M}=39.67, \mathrm{SD}=9.87$; $\mathrm{CB}$ instruction STICSA: $37.67, \mathrm{SD}=10.12$; see Fig. 1).

\section{Ratings}

Participants rated the sound stimulus as aversive $(M=2.32$ $S D=1.24$, range $1-5$, where $1=$ very negative and $9=$ very positive) and arousing $(M=6.61, S D=1.91$, range $1-9$ where $1=$ calm and $9=$ excited).

Participants displayed higher expectancy ratings of the sound with the CS+ versus CS- during acquisition [Stimulus, $F(1,90)=224.302, p<0.001]$ and extinction [Stimulus, $F(1,209.833)=146.356, p<0.001$; see Table 1 and Fig. 2]. In the early part of extinction, participants displayed higher expectancy ratings of the sound with the CS+ versus CS-, $p=0.001$. However, during late extinction, the expectancy rating of the sound with the $\mathrm{CS}+$ dropped and was similar to the CS,$- p=0.600$ [Time, $F(1,209.833)=13.566, p<0.001$; Stimulus $\times$ Time, $F(1,209.833)=17.760, p<0.001]$.

During extinction, individuals scoring higher in STICSA tended to have greater expectancy of the sound with the $\mathrm{CS}+(M=4.54, S E=0.33)$ versus $\mathrm{CS}-(M=1.92$, $S E=0.28), p<0.001$, relative to individuals lower in STICSA: CS $+(M=3.24, S E=0.33), C S-(M=1.90$, $S E=0.28), p<0.001 \quad$ [Stimulus $\times$ STICSA, $F(1$, $209.833)=7.954, p=0.006]$. Moreover, individuals with higher STICSA scores showed a similar pattern of expectancy for the sound with the CS+vs. CS- during extinction, regardless of instruction type, $p \mathrm{~s}<0.001$ [Stimulus $\times$ Instruction Type $\times$ STICSA, $F(1,209.833)=6.165$, $p=0.014]$ (No instruction CS $+: M=4.41, S E=0.47$; No instruction CS-: $M=2.28, S E=0.39$; CB instruction $\mathrm{CS}+: M=4.67, S E=0.48$; $\mathrm{CB}$ instruction $\mathrm{CS}-$ : $M=1.56, S E=0.40$ ). However, individuals with lower STICSA scores who were in the CB instruction condition displayed lower expectancy of the sound with the CS+vs. 

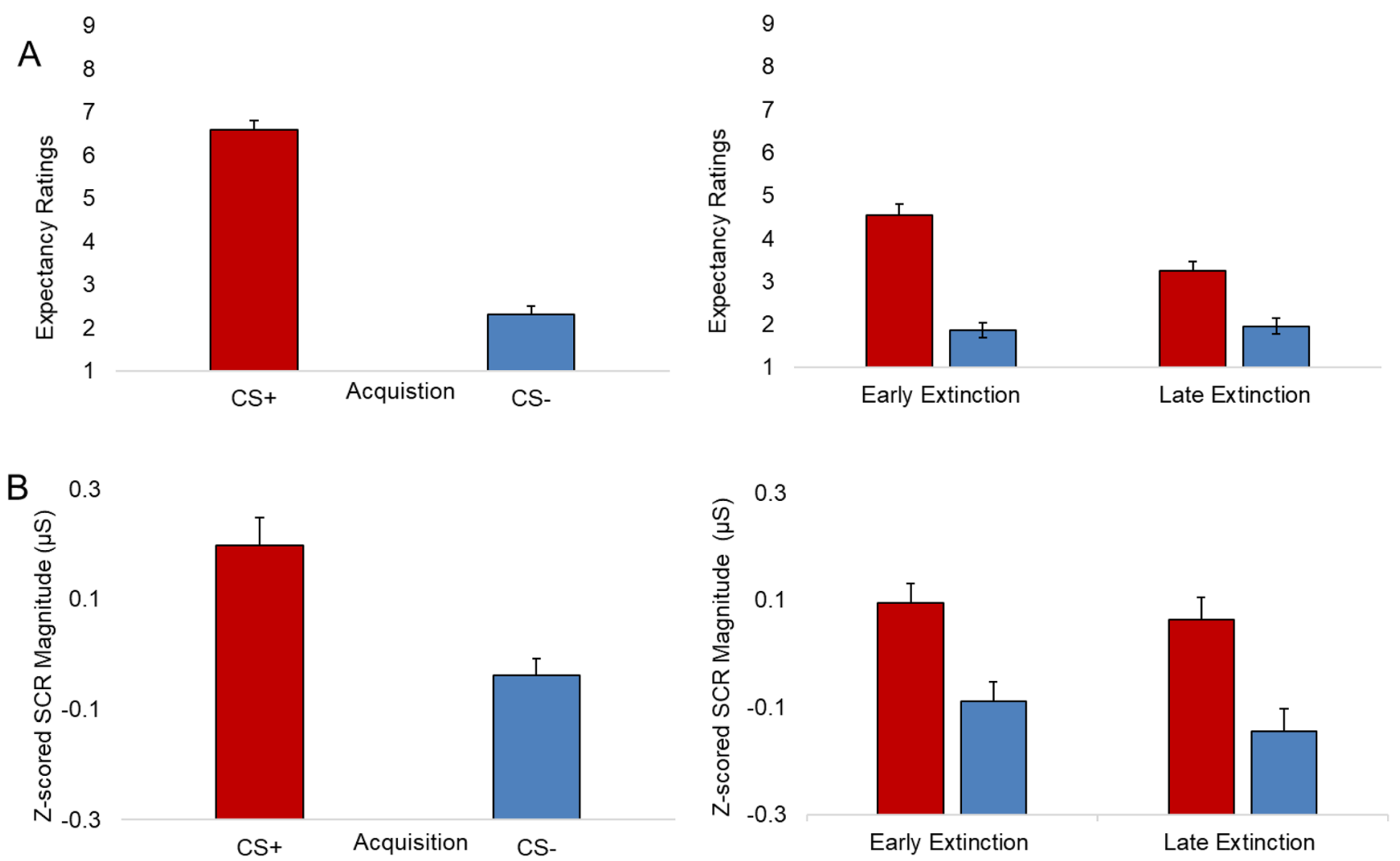

C
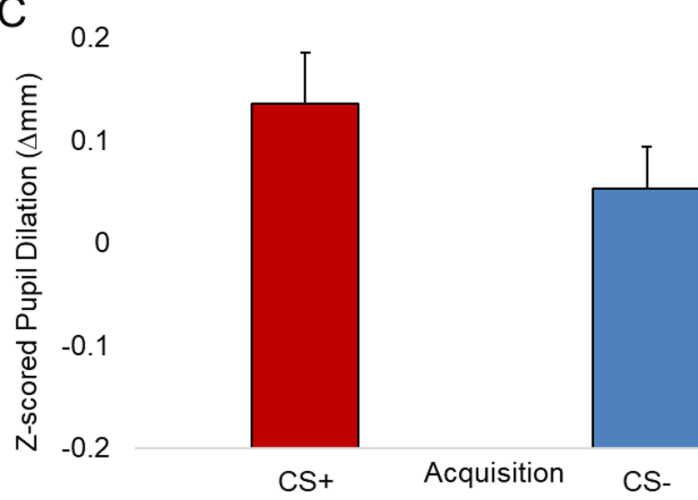

0.2

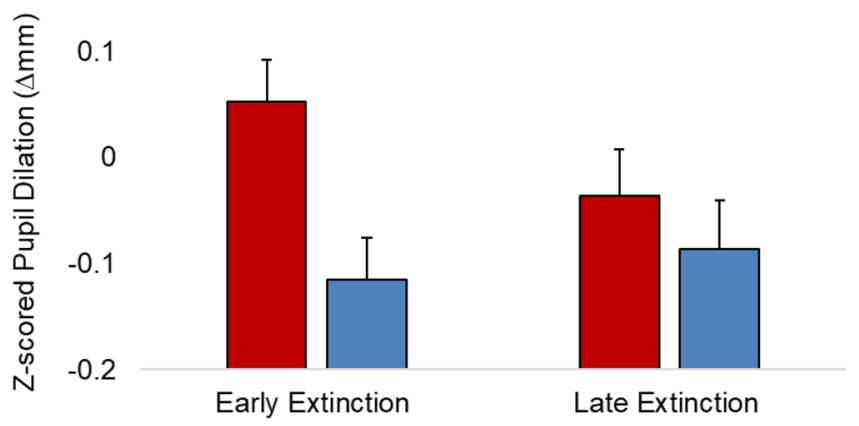

CS+ CS-

Fig. 2 Bar graph depicting mean expectancy ratings (a), SCR magnitude (b) and pupil dilation (c) during acquisition and extinction. Error bars represent standard error. Expectancy ratings, $1=$ Don't expect, $9=$ Do expect. Square root transformed and Z-scored SCR magnitude $(\mu \mathrm{S})$, skin conductance magnitude measured in microSiemens.
Z-scored pupil dilation $(\Delta \mathrm{mm})$ measured in delta millimetres. Note that the z-scoring was performed within-subjects, across acquisition and extinction, thus explaining the negative values for a number of conditions
$\mathrm{CS}$ at trend-, $p=0.060$ (No instruction $\mathrm{CS}+: M=3.88$, $S E=0.52$; No instruction $\mathrm{CS}-: M=2.61, S E=0.43$; $\mathrm{CB}$ instruction $\mathrm{CS}+: M=2.61, S E=0.43$; $\mathrm{CB}$ instruction CS-: $M=1.92, S E=0.36)$.
No other significant main effects of Instruction Type or interactions with STICSA or IU were found, max $F=2.086$. 


\section{SCR Magnitude}

Greater SCR magnitude was found for the CS+, compared to the CS- during acquisition [Stimulus, $F(1$, $92)=15.781, p<0.001$ ] and extinction [Stimulus, $F(1$, $360.769)=26.975, p<0.001$; see Table 1 and Fig. 2].

No other significant main effects of Instruction Type, Time, or interactions with IU or STICSA were found, $\max F=1.258$.

\section{Pupil Dilation}

Greater pupil dilation was observed for the $\mathrm{CS}+$, compared to the $\mathrm{CS}-$ during acquisition, but this difference was not significant [Stimulus, $F(1,89)=2.343, p=0.129$ ]. Pupil dilation was significantly larger to the CS+vs. CS- during extinction [Stimulus, $F(1,349.925)=7.061$, $p=0.008$; see Table 1 and Fig. 2].

No other significant main effects of Instruction Type, Time, or interactions with IU or STICSA were found, max $F=2.733$.

\section{Post Hoc Analyses}

We did not find effects of IU and STICSA when they were entered together into the MLMs for SCR magnitude and pupil dilation during extinction. To examine whether this was due to the measures accounting for shared variance, we conducted further analyses with IU and STICSA, where they were entered alone into the MLMs for SCR magnitude and pupil dilation.

When IU and STICSA were entered alone into the MLMs for SCR magnitude there were significant interactions [Stimulus $x$ Instruction Type $\times$ IU: $F(1,329.069)=6.322, p=0.012$; Stimulus $x$ Instruction Type $\times$ STICSA: $F(1,330.567)=6.080, p=0.014$; see Fig. 3]. These interactions were driven by larger SCR magnitude to the CS+ vs. $\mathrm{CS}-$ in individuals with lower and higher IU/STICSA [at trend for IU, $p=0.067$; at trend for STICSA, $p=0.091]$ in the no instruction condition and individuals with higher IU/STICSA in the CB instruction condition, $p \mathrm{~s}<0.01$. Only individuals with lower IU/ STICSA in the CB instruction condition displayed no significant difference in SCR magnitude for the CS+ and $\mathrm{CS}-$ during extinction, $p \mathrm{~s}>0.2$.

No other significant interactions with IU or STICSA were found for SCR magnitude and pupil dilation, $\max F=2.456$.

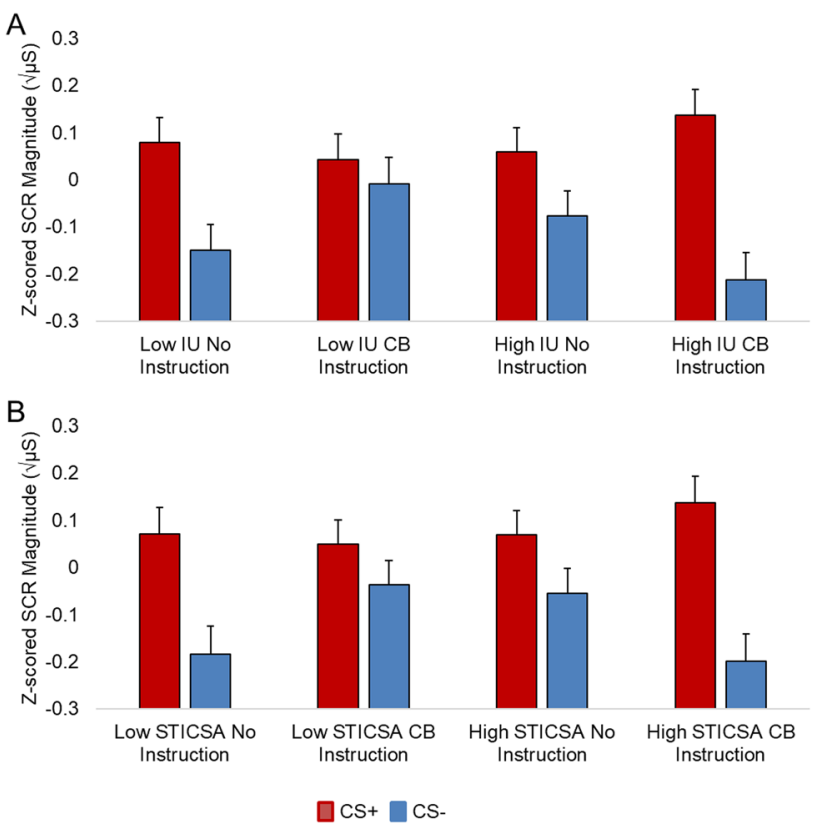

Fig. 3 SCR magnitude results during threat extinction based on IU (a) and STICSA (b) scores estimated \pm 1 standard deviation from the mean. Only individuals with lower IU/STICSA in the CB instruction group displayed no differential SCR magnitude between the CS+ and CS-. Bars represent standard error. Square root transformed and $\mathrm{Z}$-scored SCR magnitude $(\mu \mathrm{S})$, skin conductance magnitude measured in microSiemens. Note that the z-scoring was performed within-subjects, across both acquisition and extinction, thus explaining the negative values for a number of conditions

\section{Discussion}

Here we examined the effect of CBT based instructions on safety learning in individuals with varying levels of IU and STICSA. We show that the CB instruction was effective in promoting safety learning in individuals with lower IU/ STICSA. However, CB instructions were observed as ineffective in promoting safety learning for individuals with higher IU/STICSA, who continued to respond to cues previously associated with threat. This work provides a starting point for understanding the role of different self-reported measures of anxiety on basic CBT principles and safety learning, which will have relevance for future treatment targets of anxiety and stress disorders.

We observed typical patterns of conditioning in the acquisition and extinction phases, where larger SCR magnitude, pupil dilation (at trend) and expectancy ratings were found for the learned threat vs. safety cues. However, we only observed a reduction in responding to the learned threat vs. safety cues across the extinction phase for the expectancy ratings. The lack of reduction in responding across the extinction phase for SCR magnitude and pupil dilation suggests that participants did not learn the new safety association. Such effects may have occurred for the physiological 
measures during extinction because of the use of a partial reinforcement schedule during acquisition, which is known to slow down extinction (Leonard 1975).

We conducted MLMs with both IU and STICSA entered as continuous predictor variables per the preregistered analysis plan. However, this analysis yielded no significant relationships between IU, stimulus and instruction type for SCR magnitude during extinction. However, post hoc MLMs with IU and STICSA entered separately, revealed that both self-report measures interacted with stimulus and instruction type for SCR magnitude during extinction. IU and STICSA were found to influence SCR magnitude in the same way. Individuals with: (1) lower and higher self-reported anxiety in the no instruction condition, and (2) higher self-reported anxiety in the CB instruction condition displayed larger SCR magnitude to the learned threat vs. safety cues during extinction. Only individuals with lower self-reported anxiety in the $\mathrm{CB}$ instruction condition displayed no difference in SCR magnitude for the learned threat and safety cues during extinction, suggesting successful instruction-cued safety learning.

Based on previous findings (Morriss et al. 2015, 2016; Morriss 2019; Morriss and van Reekum 2019), we expected: (1) larger SCR magnitude responding for the learned threat vs. safety cues during extinction for individuals with high IU in the no instruction condition, and (2) no differences in SCR magnitude responding for the learned threat vs. safety cues during extinction for individuals with low IU in the no instruction condition. Our predictions were partially supported in that individuals with higher IU in the no instruction condition showed larger SCR magnitude for the learned threat vs. safety cues. However, this effect was not specific to IU, as a similar effect was found for STICSA. The lack of extinction for individuals with lower IU (and STICSA) in the no instruction condition was unexpected and may have occurred due to differences in task instructions. In the no instruction condition, we asked participants to 'Please pay attention to the squares and sounds' before extinction. Whilst our instruction was relatively general, previous research has shown that more direct contingency instructions speed up and maintain conditioning (for review see, Mertens et al. 2018). Therefore, our instruction may have encouraged individuals with lower IU (and STICSA) to attend more, given the suggested potential for a sound.

Individuals with higher IU/STICSA in the CB instruction condition showed larger SCR magnitude to the learned threat vs. safety cues during extinction, suggesting a lack of instruction-cued safety learning. These results for higher IU/STICSA in the CB instruction condition are similar to past findings from individuals with high IU who received no instructions (Dunsmoor et al. 2015; Lucas et al. 2018; Morriss et al. 2015, 2016; Morriss and van Reekum 2019). In a recent study, it was shown that giving direct contingency instructions before threat extinction (i.e. providing certainty that the associated square would not be followed by a sound) enhanced safety learning in individuals with high IU (Morriss and van Reekum 2019). Based on these findings, we can speculate that individuals with higher IU/STICSA in the CB instruction condition may have not immediately benefitted from the instruction because it encouraged them to work out the contingencies for themselves, rather than provide them with certainty about the contingencies.

Individuals with low IU/STICSA in the CB instruction condition displayed no difference in SCR magnitude for the learned threat and safety cues during extinction, suggesting successful safety learning. It is unclear whether the safety learning effects for low IU/STICSA in the CB instruction condition would be superior to individuals with low IU/STICSA who underwent a standard extinction (i.e. no instructions at all). Nevertheless, we can conclude that individuals with lower self-reported anxiety benefited from the CB instruction instructions, compared to individuals with higher self-reported anxiety. Whilst we only examined a single session of extinction with simplified instructions, these results highlight that individuals with higher selfreported anxiety may need more practise and intense CBT with exposure therapy than their lower self-reported anxiety counterparts.

In previous work, IU has been found to be specifically related, over self-reported trait anxiety and worry measures, to differences in SCR magnitude (and other readout measures) during extinction (Dunsmoor et al. 2015; Lucas et al. 2018; Morriss 2019; Morriss and van Reekum 2019; Morriss et al. 2015, 2016). In the current study, the results from the MLMs with IU and STICSA entered as continuous predictors suggested no specificity of IU and STICSA for SCR magnitude differences during extinction. Yet further MLMs where IU/STICSA were entered as grouping factors suggested more specificity of IU over STICSA for SCR magnitude (see Supplementary Material). Notably, to our knowledge, this is the first time IU has been pitted against STICSA. It may be that STICSA is also a reliable self-report measure for examining anxiety and threat extinction. However, further replication work is needed to ascertain if there is any specificity or equality between the IU and STICSA measures.

Interestingly, the results differed depending on the type of measurement we used. In line with past research on IU and threat extinction (Dunsmoor et al. 2015; Lucas et al. 2018; Morriss et al. 2015, 2016, 2018b), we found significant relationships between IU and skin conductance response. However, we did not find IU-related effects for pupil dilation during extinction. From this, we speculate that skin conductance, compared to pupil dilation, may be a more robust measure for examining individual differences in IU within an extinction context. While both skin conductance and pupil 
dilation are under the control of the sympathetic branch of the autonomic nervous system (Dawson et al. 2000) there may be differences in the rate of habituation, as well as tonic and phasic activity. For the ratings we observed results with STICSA, where individuals scoring higher in STICSA relative to lower in STICSA tended to have higher ratings of expectancy of the sound with the CS+ versus CS-, regardless of instruction condition. In a previous extinction experiment, similar effects were observed on the expectancy ratings with self-reported trait anxiety (STAI) (Morriss et al. 2019a, b). Thus, both self-reported STICSA and STAI, compared to IU, may be closely aligned to more conscious processes such as self-reported expectancy. The difference in relationships between self-reported IU and trait anxiety with other behavioural measures supports current theory which postulates that trait anxiety (STICSA or STAI) is a higher order factor (i.e., more consciously accessible), whilst IU is lower order factor (i.e., less consciously accessible) (for further discussion see Carleton 2016b).

A limitation of the current study is that there was no manipulation check that the different instruction types worked. However, with instruction-based studies of threat extinction, such formal manipulation checks tend not to be used. Instructions are presumed to work if there are differences between conditions based on instruction type. In this study, only differences in SCR magnitude based on IU/ STICSA and instruction type were found. Therefore, in this study the instruction manipulation may have been weak. These findings highlight the need for the development of manipulation checks in future instruction-based studies of threat extinction, such as contingency awareness.

In conclusion, these initial results provide some insight into how simple CB instructions combined with exposure are applied differently in individuals with varying levels of self-reported anxiety. Tentatively, the current findings suggest that individuals with lower IU/STICSA benefit from a brief CBT like intervention, whilst individuals with higher IU/STICSA do not. Further experimental and clinical research is needed to assess how combined CBT with exposure therapy can promote safety learning in individuals with higher levels of self-reported anxiety.

Acknowledgements The researchers would like to thank Laura Bucher, Charlotte Elizabeth and Martina Mutti for their help in data collection and preprocessing.

Funding The funding was provided by National Alliance for Research on Schizophrenia and Depression (27567), Economic and Social Research Council (ES/L010119/1) and Economic and Social Research Council (ES/R01145/1).

\section{Compliance with Ethical Standards}

Conflict of Interest The dataset reported here is not part of any published or currently in press works. Shannon Wake, Carien M. van Reekum, Helen Dodd and Jayne Morriss have no conflict of interests to declare.

Open Access This article is licensed under a Creative Commons Attribution 4.0 International License, which permits use, sharing, adaptation, distribution and reproduction in any medium or format, as long as you give appropriate credit to the original author(s) and the source, provide a link to the Creative Commons licence, and indicate if changes were made. The images or other third party material in this article are included in the article's Creative Commons licence, unless indicated otherwise in a credit line to the material. If material is not included in the article's Creative Commons licence and your intended use is not permitted by statutory regulation or exceeds the permitted use, you will need to obtain permission directly from the copyright holder. To view a copy of this licence, visit http://creativecommons.org/licenses/by/4.0/.

\section{References}

Ben-Shakhar, G. (1985). Standardization within individuals: A simple method to neutralize individual differences in skin conductance. Psychophysiology, 22(3), 292-299.

Benjamini, Y., \& Hochberg, Y. (1995). Controlling the false discovery rate: a practical and powerful approach to multiple testing. Journal of the Royal Statistical Society: Series B (Methodological), 57(1), 289-300.

Blechert, J., Michael, T., Vriends, N., Margraf, J., \& Wilhelm, F. H. (2007). Fear conditioning in posttraumatic stress disorder: Evidence for delayed extinction of autonomic, experiential, and behavioural responses. Behaviour Research and Therapy, 45(9), 2019-2033.

Bomyea, J., Ramsawh, H., Ball, T. M., Taylor, C. T., Paulus, M. P., Lang, A. J., et al. (2015). Intolerance of uncertainty as a mediator of reductions in worry in a cognitive behavioral treatment program for generalized anxiety disorder. Journal of Anxiety Disorders, $33,90-94$.

Boswell, J. F., Thompson-Hollands, J., Farchione, T. J., \& Barlow, D. H. (2013). Intolerance of uncertainty: A common factor in the treatment of emotional disorders. Journal of Clinical Psychology, 69, 630-645.

Bouton, M. E. (2002). Context, ambiguity, and unlearning: Sources of relapse after behavioral extinction. Biological Psychiatry, 52(10), 976-986.

Carleton, R. N. (2016a). Fear of the unknown: One fear to rule them all? Journal of Anxiety Disorders, 41, 5-21.

Carleton, R. N. (2016b). Into the unknown: A review and synthesis of contemporary models involving uncertainty. Journal of Anxiety Disorders, 39, 30-43.

Craske, M. G., Treanor, M., Conway, C. C., Zbozinek, T., \& Vervliet, B. (2014). Maximizing exposure therapy: An inhibitory learning approach. Behaviour Research and Therapy, 58, 10-23.

Dawson, M. E., Schell, A. M., \& Filion, D. L. (2000). The electrodermal system. In J. T. Cacioppo, L. G. Tassinary, \& G. G. Berntson (Eds.), Handbook of physiology (2nd ed., pp. 200-223). Cambridge, UK: Cambridge University Press.

Dugas, M. J., Brillon, P., Savard, P., Turcotte, J., Gaudet, A., Ladoucuer, R., et al. (2010). A randomized clinical trial of cognitive-behavioral therapy and applied relaxation for adults with generalized anxiety disorder. Behavior Therapy, 41, 46-58. 
Dugas, M. J., \& Ladouceur, R. (2000). Treatment of GAD: Targeting intolerance of uncertainty in two types of worry. Behavior Modification, 24, 635-657.

Dugas, M. J., Ladouceur, R., Léger, E., Freeston, M. H., Langolis, F., Provencher, M. D., et al. (2003). Group cognitive-behavioral therapy for generalized anxiety disorder: Treatment outcome and long-term follow-up. Journal of Consulting and Clinical Psychology, 71, 821-825.

Dugas, M. J., \& Robichaud, M. (2007). Cognitive-behavioural treatment for generalized anxiety disorder. New York, NY: Taylor \& Francis Group.

Dunsmoor, J. E., Campese, V. D., Ceceli, A. O., LeDoux, J. E., \& Phelps, E. A. (2015). Novelty-facilitated extinction: Providing a novel outcome in place of an expected threat diminishes recovery of defensive responses. Biological Psychiatry, 78(3), 203-209.

Freeston, M. H., Rhéaume, J., Letarte, H., Dugas, M. J., \& Ladouceur, R. (1994). Why do people worry? Personality and Individual Differences, 17(6), 791-802.

Grady, A. K., Bowen, K. H., Hyde, A. T., Totsch, S. K., \& Knight, D. C. (2016). Effect of continuous and partial reinforcement on the acquisition and extinction of human conditioned fear. Behavioral Neuroscience, 130(1), 36.

Grös, D. F., Antony, M. M., Simms, L. J., \& McCabe, R. E. (2007). Psychometric properties of the State-trait inventory for cognitive and somatic anxiety (STICSA): Comparison to the Statetrait anxiety inventory (STAI). Psychological Assessment, 19(4), 369

Hewitt, S. N., Egan, S., \& Rees, C. (2009). Preliminary investigation of intolerance of uncertainty treatment for anxiety disorders. Clinical Psychologist, 13, 52-58.

Hofmann, S. G. (2008). Cognitive processes during fear acquisition and extinction in animals and humans: Implications for exposure therapy of anxiety disorders. Clinical Psychology Review, 28(2), 199-210.

Knowles, K. A., \& Olatunji, B. O. (2019). Enhancing inhibitory learning: The utility of variability in exposure. Cognitive and Behavioral Practice, 26(1), 186-200.

Leonard, D. W. (1975). Partial reinforcement effects in classical aversive conditioning in rabbits and human beings. Journal of Comparative and Physiological Psychology, 88(2), 596.

Leuchs, L., Schneider, M., \& Spoormaker, V. I. (2019). Measuring the conditioned response: A comparison of pupillometry, skin conductance, and startle electromyography. Psychophysiology, 56(1), e13283.

Lonsdorf, T. B., Menz, M. M., Andreatta, M., Fullana, M. A., Golkar, A., Haaker, J., et al. (2017). Don't fear 'fear conditioning': Methodological considerations for the design and analysis of studies on human fear acquisition, extinction, and return of fear. Neuroscience \& Biobehavioral Reviews, 77, 247-285.

Lucas, K., Luck, C. C., \& Lipp, O. V. (2018). Novelty-facilitated extinction and the reinstatement of conditional human fear. Behaviour Research and Therapy, 109, 68-74.

McEvoy, P. M., \& Erceg-Hurn, D. M. (2015). The search for universal transdiagnostic and trans-therapy change processes: Evidence for intolerance of uncertainty. Journal of Anxiety Disorders, 41, 96-107.

Mertens, G., Boddez, Y., Sevenster, D., Engelhard, I. M., \& De Houwer, J. (2018). A review on the effects of verbal instructions in human fear conditioning: Empirical findings, theoretical considerations, and future directions. Biological Psychology, 137, 49-64.

Michael, T., Blechert, J., Vriends, N., Margraf, J., \& Wilhelm, F. H. (2007). Fear conditioning in panic disorder: Enhanced resistance to extinction. Journal of Abnormal Psychology, 116(3), 612.

Milad, M. R., Orr, S. P., Lasko, N. B., Chang, Y., Rauch, S. L., \& Pitman, R. K. (2008). Presence and acquired origin of reduced recall for fear extinction in PTSD: Results of a twin study. Journal of Psychiatric Research, 42(7), 515-520.

Milad, M. R., Pitman, R. K., Ellis, C. B., Gold, A. L., Shin, L. M., Lasko, N. B., et al. (2009). Neurobiological basis of failure to recall extinction memory in posttraumatic stress disorder. Biological Psychiatry, 66(12), 1075-1082.

Milad, M. R., \& Quirk, G. J. (2012). Fear extinction as a model for translational neuroscience: Ten years of progress. Annual Review of Psychology, 63, 129-151.

Morriss, J. (2019). What do I do now? Intolerance of uncertainty is associated with discrete patterns of anticipatory physiological responding to different contexts. Psychophysiology, 56, e13396.

Morriss, J., Chapman, C., Tomlinson, S., \& Van Reekum, C. M. (2018a). Escape the bear and fall to the lion: The impact of avoidance availability on threat acquisition and extinction. Biological Psychology, 138, 73-80.

Morriss, J., Christakou, A., \& Van Reekum, C. M. (2015). Intolerance of uncertainty predicts fear extinction in amygdala-ventromedial prefrontal cortical circuitry. Biology of Mood \& Anxiety Disorders, 5(1), 1.

Morriss, J., Christakou, A., \& Van Reekum, C. M. (2016). Nothing is safe: Intolerance of uncertainty is associated with compromised fear extinction learning. Biological Psychology, 121, 187-193.

Morriss, J., McSorley, E., \& Van Reekum, C. M. (2018b). I don't know where to look: The impact of intolerance of uncertainty on saccades towards non-predictive emotional face distractors. Cognition and Emotion, 32(5), 953-962.

Morriss, J., Saldarini, F., Chapman, C., Pollard, M., \& van Reekum, C. M. (2019b). Out with the old and in with the new: The role of intolerance of uncertainty in reversal of threat and safety. Journal of Experimental Psychopathology, 10(1), 1-11.

Morriss, J., Saldarini, F., \& Van Reekum, C. M. (2019a). The role of threat level and intolerance of uncertainty in extinction. International Journal of Psychophysiology, 142, 1-9.

Morriss, J., \& van Reekum, C. (2019). I feel safe when I know: Contingency instruction promotes threat extinction in high intolerance of uncertainty individuals. Behaviour, Research and Therapy, 116, $111-118$.

Neumann, D. L., \& Waters, A. M. (2006). The use of an unpleasant sound as an unconditional stimulus in a human aversive Pavlovian conditioning procedure. Biological Psychology, 73(2), 175-185.

Peugh, J. L. (2010). A practical guide to multilevel modeling. Journal of School Psychology, 48(1), 85-112.

Ree, M. J., French, D., MacLeod, C., \& Locke, V. (2008). Distinguishing cognitive and somatic dimensions of state and trait anxiety: Development and validation of the State-trait inventory for cognitive and somatic anxiety (STICSA). Behavioural and Cognitive Psychotherapy, 36(3), 313-332.

Robichaud, M., Koerner, N., \& Dugas, M. J. (2019). Cognitive behavioral treatment for generalized anxiety disorder: From science to practice. Routledge.

Shihata, S., McEvoy, P. M., Mullan, B. A., \& Carleton, R. N. (2016). Intolerance of uncertainty in emotional disorders: What uncertainties remain? Journal of Anxiety Disorders, 41, 115-124. https:// doi.org/10.1016/j.janxdis.2016.05.001.

Shin, L. M., \& Liberzon, I. (2009). The neurocircuitry of fear, stress, and anxiety disorders. Neuropsychopharmacology, 35(1), 169-191.

Simos, G., \& Hofmann, S. G. (2013). CBT for anxiety disorders a practitioner book. West Sussex, UK: Wiley.

Snijders, T. A. (2005). Power and sample size in multilevel linear models. Encyclopedia of Statistics in Behavioral Science, 3 , 1570-1573.

van der Heiden, C., Muris, P., \& van der Molen, H. T. (2012). Randomized controlled trial on the effectiveness of metacognitive 
therapy and intolerance- of-uncertainty therapy for generalized anxiety disorder. Behaviour Research and Therapy, 50, 100-109.

Xia, W., Dymond, S., Lloyd, K., \& Vervliet, B. (2017). Partial reinforcement of avoidance and resistance to extinction in humans. Behaviour Research and Therapy, 96, 79-89.
Publisher's Note Springer Nature remains neutral with regard to jurisdictional claims in published maps and institutional affiliations. 